\title{
The Neuroprotective Effect of Irisin in Ischemic Stroke
}

\author{
Yaqiang Liu, Chunhua Zhu*, Jiahui Guo, Yonghong Chen and Chaoyue Meng \\ Department of Neurology, Second Hospital of Hebei Medical University, Shijiazhuang, China
}

Irisin is a PGC-1 $\alpha$-dependent myokine that causes increased energy expenditure by driving the development of white adipose tissue into brown fat-like tissue. Exercise can improve irisin levels and lead to its release into the blood. In ischemic stroke, neurons are always sensitive to energy supply; after a series of pathophysiological processes, reactive oxygen species that are detrimental to cell survival via mitochondrial dysfunction are generated in large quantities. As a protein associated with exercise, irisin can alleviate brain injury in the pathogenesis of ischemic stroke. It is thought that irisin can upregulate the levels of brain-derived neurotrophic factor (BDNF), which protects nerve cells from injury during ischemic stroke. Furthermore, the release of irisin into the blood via exercise influences the mitochondrial dynamics crucial to maintaining the normal function of nerve cells. Consequently, we intended to summarize the known effects of irisin during ischemic stroke.

Keywords: exercise, stroke, AMPK, mitochondrial dynamics, irisin

\section{OPEN ACCESS}

Edited by:

Gang Chen,

First Affiliated Hospital of Soochow

University, China

Reviewed by:

Xinchun Jin,

Capital Medical University, China Johannes Boltze,

University of Warwick,

United Kingdom

*Correspondence:

Chunhua Zhu

zhuchunhua78@sina.com

Received: 30 July 2020 Accepted: 01 December 2020 Published: 22 December 2020

Citation:

Liu Y, Zhu C, Guo J, Chen Y and Meng C (2020) The Neuroprotective Effect of Irisin in Ischemic Stroke.

Front. Aging Neurosci. 12:588958. doi: 10.3389/fnagi.2020.588958

\section{INTRODUCTION}

The incidence of stroke has increased rapidly over the past few decades, causing it to become one of the main causes of death and long-term disability worldwide (Huang et al., 2012; Katan and Luft, 2018). Especially in low- and middle-income countries, the incidence of stroke-related mortality is increasing, resulting in a high economic burden for both the patients and society (Karimi-Khouzani et al., 2017). Ischemic stroke accounts for $\sim 80 \%$ of stroke cases (Lapchak and Zhang, 2017). Among all types of ischemic stroke, focal ischemic stroke is the most common. Focal ischemic stroke is caused by middle cerebral artery occlusion (MCAO) (permanent or transient), resulting in a lack of blood flow through the MCA. Defective blood supply can cause a shortage of glucose and oxygen in nerve cells, thus increasing reactive oxygen species (ROS) production and disrupting cell homeostasis. These complications lead to pathophysiological processes including excitotoxicity, oxidative stress, inflammation, apoptosis, and cell death (Khoshnam et al., 2017).

Mitochondria play a pivotal role in the pathophysiology of cerebral ischemic reperfusion. As highly dynamic organelles, mitochondria undergo morphological transformation through fission and fusion. In ischemic stroke, fission and fusion play critical roles in maintaining mitochondrial homeostasis when nerve cells lose blood supply. When mitochondria are damaged, fusion exerts a protective effect, allowing functional mitochondria to complement dysfunctional mitochondria through combining components between organelles. Fission is needed to create new mitochondria. However, excessive fission results in mitochondrial dysfunction (Li and Liu, 2018; Wang et al., 2020). In ischemic stroke, mitochondria are the main source of ROS. As a source of stress, excessive ROS damages the normal morphology of mitochondria, disrupting brain cells ( $\mathrm{Li}$ and Liu, 2018). Therefore, maintaining mitochondrial integrity can serve as an alternative candidate for the development of neuroprotective tactics for treating cerebral ischemic injury. 
Physical exercise can reportedly alleviate some of these pathophysiological processes. In addition, in the rehabilitation stage of stroke, exercise can also effectively improve sequelae symptoms (Li et al., 2017; Ryan et al., 2017). In 2013, studies reported that brain-derived neurotrophic factor (BDNF) is a possible mediator of the neurological benefits of exercise (Mang et al., 2013). BDNF is an abundant growth factor that is correlated with activity-induced neuroplasticity (Mang et al., 2013), and is upregulated by exercise in the animal brain (Berchtold et al., 2005; Rasmussen et al., 2009; Quirié et al., 2012). In a chronic stroke, treadmill high-intensity interval training elicited a significantly acute increase in BDNF (Boyne et al., 2019). Vascular endothelial growth factor (VEGF) is another neurotrophin that accumulates in human blood during exercise (Wahl et al., 2014). Peripheral increases in VEGF expression promotes perilesional angiogenesis and neurologic recovery in animal models of post-acute stroke (Zhang et al., 2000; Yang et al., 2010). Furthermore, several studies are substantiating the benefits of an exercise intervention on induced brain injury in animal stroke models (Ding et al., 2005; Matsuda et al., 2011; Sakakima et al., 2012; Otsuka et al., 2016). A recent study also demonstrated that the exercise-induced hormone irisin contributes to the neuroprotective effect of physical exercise against cerebral ischemia (Li et al., 2017).

The previously unknown hormone irisin was discovered by Boström et al. (2012). Irisin is released into the blood through the enzymatic hydrolysis of PGC- $1 \alpha$ after exercise, which could stimulate the transformation from mouse and human white fat cells into brown fat cells (Figure 1) (Boström et al., 2012). Since then, several studies have confirmed that irisin plays a protective role in the pathogenesis of many diseases, including neurodegenerative diseases, such as Alzheimer's disease, and cardiovascular diseases (Jin et al., 2018; Kim et al., 2018; Clark and Vissel, 2019; Conti et al., 2019; Young et al., 2019; Zhao et al., 2019). However, as research on the role of irisin in ischemic stroke is still limited, it is necessary to further elucidate its activity.

This review intends to summarize the structure and distribution of irisin in different tissues, the neuroprotective effect of irisin generation via exercise in ischemic stroke, and the correlation of exercise and irisin on mitochondrial dynamics. We, therefore, aim to provide a new research direction for exploring new treatment methods for ischemic stroke.

\section{STRUCTURE AND DISTRIBUTION OF IRISIN}

Irisin was originally reported as a new hormone secreted from muscle cells upon exercise. It is encoded by $P G C-1 \alpha$, which is involved in many pathways related to energy metabolism (Boström et al., 2012). PGC-1 $\alpha$ can stimulate skeletal muscle cells to express FNDC5 (a signal peptide with 29 amino acid residues), type III fibronectin assembly with 112 amino acid residues, and a C-terminal transmembrane domain with 65 amino acid residues. After the discovery of FNDC5 glycosylation by proteolytic enzymes, a new protein was identified. Irisin

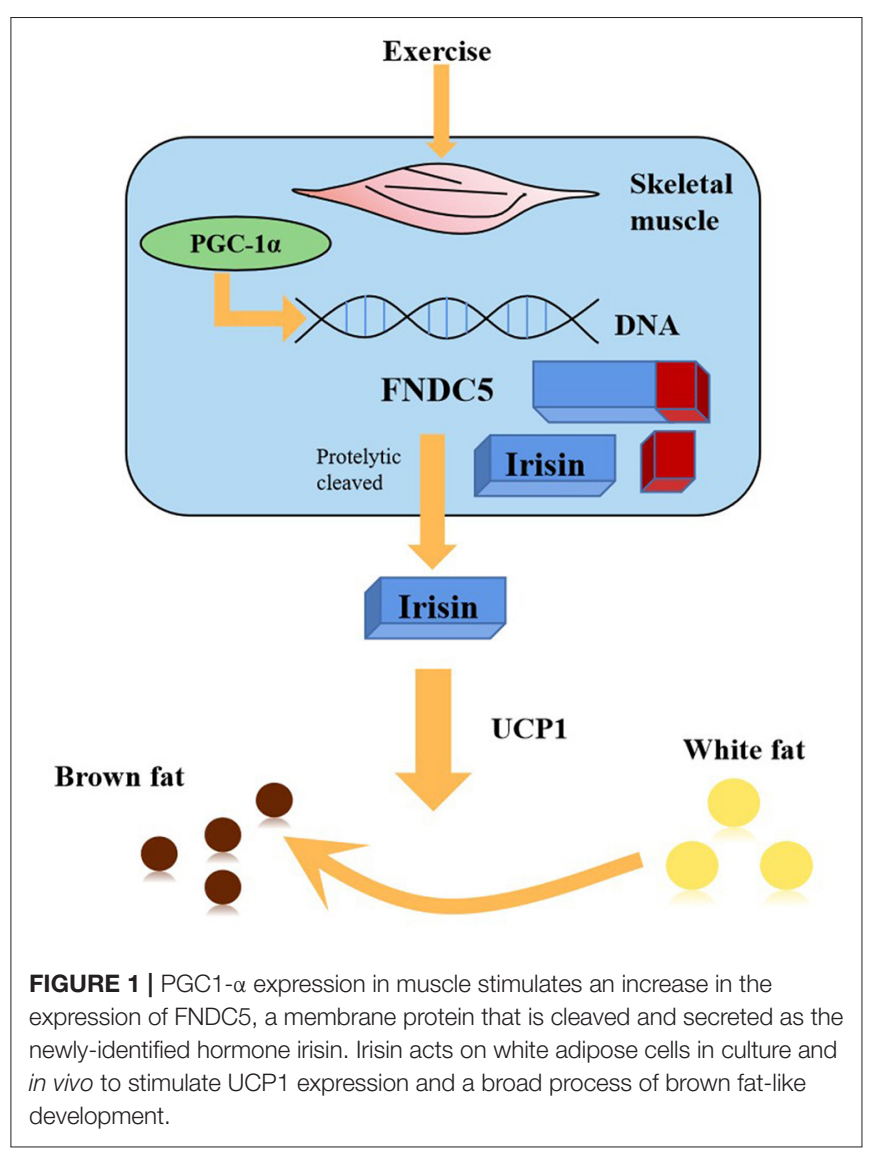

consists of 112 amino acid residues and a fibronectin III domain (Boström et al., 2012; Erickson, 2013). Previous research has revealed preliminary evidence that irisin is not only expressed in mammalian organs and tissues such as the heart, skeletal muscle, and brain (Aydin et al., 2014) but also in the thyroid, ovary, liver, lung, testis, esophagus, fat, and other tissues (Wrann et al., 2013). The distribution of irisin can also be detected in different brain regions and cell groups such as in Purkinje cells in the cerebellum (Varela-Rodríguez et al., 2016), astrocytes in the hippocampus (Piya et al., 2014), neurons in the brain (Wang et al., 2018), the hypothalamus (Dun et al., 2013), and even cerebrospinal fluid (CSF) (Aydin et al., 2013). Importantly, irisin has been demonstrated to have pivotal roles in attenuating inflammation, reducing oxidative stress, and alleviating apoptosis, as well as ameliorating mitochondrial dysfunction (Tu et al., 2020). Consequently, the discovery and distribution of irisin has provided a theoretical basis for exploring its effects in many diseases, especially in ischemic stroke.

\section{THE ROLE OF IRISIN IN STROKE}

Skeletal muscle is a crucial organ in humans, accounting for $\sim 40 \%$ of the human body weight. As the most energy-consuming organ, skeletal muscle accelerates the synthesis and secretion of muscle factors with active ingredients during exercise. These factors can act on other organs (such as the adipose tissue 
and the brain) in various ways by regulating sugar, lipid, and protein metabolism (Febbraio and Pedersen, 2005; Lee et al., 2015). Hence, the regulation of irisin/FNDC5 has obvious motor involvement.

The role of FNDC5/irisin in learning and memory is mediated by BDNF expression, which plays an important role in neural remodeling in conditions such as Alzheimer's disease (Wrann et al., 2013). A large number of studies have investigated the effect of exercise on irisin secretion. Exercise can upregulate BDNF levels in the hippocampus via PGC-1 $\alpha$ activation and FNDC5 expression (Wrann et al., 2013; Xu, 2013; Yau et al., 2015). Experts have reached a consensus that BDNF may exert a neuroprotective role via irisin expression. For example, Islam et al. (2017) demonstrated that long-term exercise could increase BDNF expression in the brain through the PGC-1 $\alpha$-FNDC5 axis. It has also been shown that BDNF can enhance neuronal survival and migration (Raefsky and Mattson, 2017). Further research based on these studies demonstrated that BDNF expression was regulated by the application of irisin to a rodent stroke model during cerebral ischemia-reperfusion (Asadi et al., 2018). This study elucidated that BDNF is a crucial regulator of the beneficial effects conferred by irisin in ischemic stroke. As a protein that leads to irisin expression, the peripheral delivery of FNDC5 to the liver via adenoviral vectors could increase the level of BDNF and other neuroprotective genes in the hippocampus (Boström et al., 2012). This implies that irisin, or other factors induced by irisin, can cross the blood-brain barrier to affect gene expression in the brain. This discovery provides a theoretical basis for exploring the effects of irisin in ischemic stroke.

A recent study has reported that irisin protects the bloodbrain barrier from ischemic injury by decreasing the expression of MMP-9 (Guo et al., 2019). Some research has demonstrated that exercise-induced irisin protects neurons from ischemiareperfusion injury by reducing the volume of cerebral infarction, brain edema, and weight loss via Akt activation, which then leads to the activation of BDNF (Croll et al., 1999) and the ERK1/2 pathways (Li et al., 2017). Consistently, a recent study has indicated that brain edema and neurological function are alleviated by irisin during cerebral ischemia-reperfusion in mice and inflammation factors such as IL- $1 \beta$ and TNF- $\alpha$ are decreased and that apoptosis is reduced in the hippocampal neurons as a result of irisin treatment via activation of the Notch signaling pathway (Jin et al., 2019). Furthermore, irisin exerts a beneficial role in vivo (Peng et al., 2017) and in vitro (Yu et al., 2020) during ischemic stroke by suppressing the ROS/NLRP3 and TLR4/MYD88 signaling pathways, respectively. Together, these results suggest that irisin may exert a neuroprotective role during an ischemic stroke (Figure 2).

Notably, although Li et al. (2017) have found that in MCAO mice, irisin expression in the plasma is negatively associated with the concentration of pro-inflammatory cytokines IL-6 and IL- $\alpha$, the transition of white adipose into brown adipose tissue may take some time, which is usually not available. Consequently, exogenous irisin treatment may be necessary during an ischemic stroke (Peng et al., 2017; Jin et al., 2019; $\mathrm{Yu}$ et al., 2020). Moreover, the patterns of exercise are very important for the generation of irisin. The forms of exercise

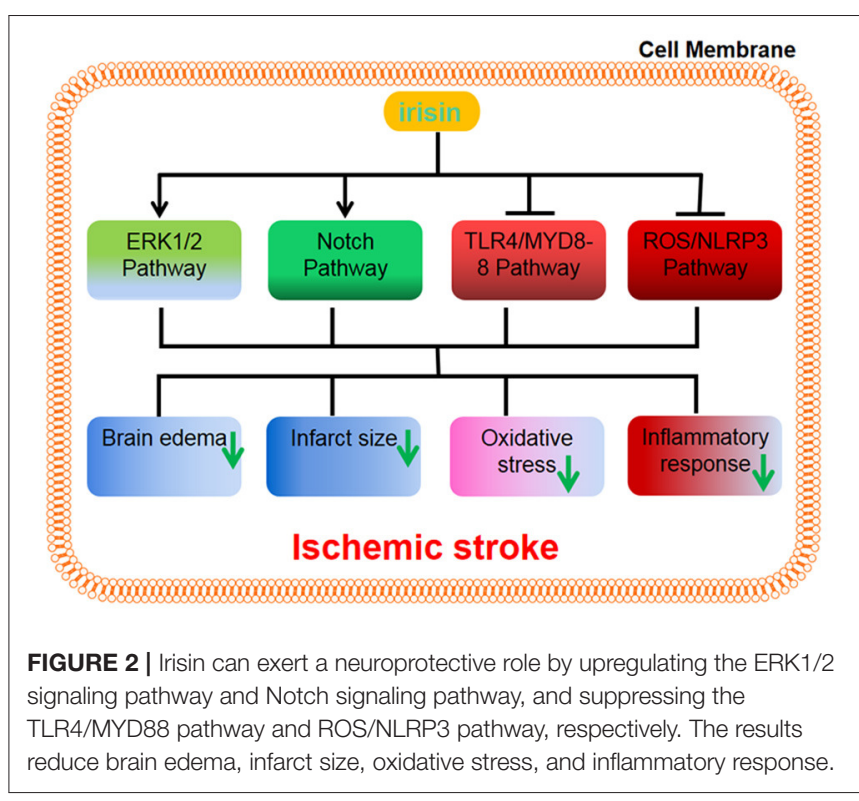

include endurance and resistance exercise training; the former is an aerobic and cardiovascular form of exercise, while the latter focuses more on muscle strength and hypertrophy (Cornelissen and Smart, 2013; Ryan et al., 2017). At present, evidence suggests that irisin is involved in endurance exercise. This is to be expected as resistance training activates the PGC- $1 \alpha$ isoform PGC-1 $\alpha 4$, while endurance exercise regulates PGC- $1 \alpha 1$, an upstream transcriptional regulator of FNDC5 (Ruas et al., 2012). Consistently, a study has also demonstrated that there is no difference in serum irisin levels between the control subjects and those who performed exercise, after the high-intensity interval and resistance training (He et al., 2018).

\section{IRISIN AND MITOCHONDRIAL DYNAMICS}

As previously described, the discovery of irisin provides an alternative direction for studying the potential treatment methods for ischemic stroke. In 2017, Lidongjie et al. found that irisin synthesis reduces the infarct volume and the degree of brain edema and improves the neurobehavioral score in an oxygenglucose deprivation model (Li et al., 2017). During physiological processes, the exercise-induced actin irisin can participate in energy metabolism by affecting mitochondrial function. Some studies have confirmed that when the regulation of energy metabolism is impaired, mitochondrial function is damaged, which further aggravates tissue damage in ischemia-reperfusion models (Chen et al., 2015; Flippo et al., 2018; Guo et al., 2018; Kim et al., 2018; Zabala et al., 2019; Zhang et al., 2019; Zhao et al., 2019). Exercise and irisin pretreatment exert a protective role by affecting the mitochondrial dynamics in tissues (Zhang et al., 2014; Chen et al., 2017; Bi et al., 2019). In addition, in the presence of ROS, exercise can maintain the normal morphology of mitochondria by activating AMPK (Trewin et al., 2018). 


\section{MITOCHONDRIAL DYNAMICS AND STROKE}

Mitochondrial dynamics mainly consist of fission and fusion. Fission is mediated by the proteins Drp1, Fis1, and MFF. Drp1 is recruited from the cytosol to the outer membrane of mitochondria and interacts with its receptor proteins MFF and Fis1 to create the fission complex. Drp1 is then oligomerized into filaments that wrap around mitochondria, leading to mitochondrial constriction and sequential separation of the inner and outer membrane. Drp1 reportedly has a crucial role in ischemic stroke; brain edema, the infarct area, and other neuronal injuries are alleviated following Drp1 downregulation (Anzell et al., 2018; Kameoka et al., 2018).

Three different GTPases mediate fusion, including Opa1 and Mfn1/2. Mfn1/2 are anchored to the outer membrane of mitochondria, while inner membrane fusion is mediated by Opa1. A lack of mitofusins prevents fusion of both the outer and inner membrane of the mitochondria, while the loss of Opal only blocks fusion of the inner membrane. Mitochondrial fusion proteins are less studied in ischemic stroke. Mfn2 is reported to exert an anti-apoptotic effect, and its expression decreases in the presence of ROS. Opal can attenuate infarct volume in ischemic stroke, and its expression is increased after exercise (Anzell et al., 2018; Kameoka et al., 2018; Wei et al., 2019; Lai et al., 2020).

In ischemic stroke, cell survival and pathobiology are involved in mitochondrial dynamics. As mitochondrial dynamics processes, fission and fusion are crucial to mitochondrial function. Fusion is presumed to be beneficial to cell survival, but studies show that fission facilitates cell death ( $\mathrm{Li}$ and Liu, 2018; Wang et al., 2020). Studies report that irisin can inhibit excessive Drp1-related mitochondrial fission and ROS, which exerts a protective role in ischemic disease (Bi et al., 2019; Tan et al., 2019). Furthermore, exercise can improve mitochondrial function in the brain by increasing the activity of the mitochondrial complex and Drp1 expression (Gusdon et al., 2017). Consequently, mitochondrial dynamics may have a key exercise-related role following ischemic stroke.

\section{AMPK AND MITOCHONDRIAL DYNAMICS}

It is reported that mitochondrial homeostasis is closely related to AMPK upregulation associated with altered cell energy metabolism (Siteneski et al., 2018). Animal studies show that irisin activates AMPK to inhibit liver cholesterol synthesis (Tang et al., 2016). Therefore, it is speculated that irisin may influence mitochondria by regulating AMPK expression.

AMPK is a heterotrimer including an $\alpha$-subunit and two regulatory subunits, $\beta$ and $\gamma$. The $\alpha$-subunit is the main catalytic part of AMPK, containing a kinase domain and the key residue Thr172. When the ratio of ATP-AMP decreases, the AMPK complex is activated by phosphorylation on Thr172 in the $\alpha$ subunit. The activated AMPK affects the mitochondrial dynamics by activating downstream substrates. When stresses, such as ischemia or hypoxia, are applied, the phosphorylated AMPK directly phosphorylates MFF. MFF then recruits Drp1 to the mitochondrial membrane, selectively causing fission of the damaged mitochondria and protecting normal mitochondrial function (Wang and Youle, 2016; Herzig and Shaw, 2018).

It has been reported that exercise is a potential activator of AMPK, demonstrating the possibility that AMPK can affect mitochondrial dynamics via exercise (Trewin et al., 2018). Moreover, irisin is necessary for mediating AMPK activity (Fan et al., 2020). Notably, AMPK reactivation can attenuate hyperglycemia-mediated mitochondrial injury. In heart ischemia, irisin can improve the expression of mitochondrial fusion proteins Opal and Mfn1 by activating the AMPK signaling pathway; blocking the AMPK pathway inhibits the regulatory activity of irisin on mitochondrial homeostasis (Fan et al., 2020). Irisin also activates the AMPK/UCP2 signaling pathway, which exerts a protective role on ischemia/reperfusioninduced renal injury (Zhang et al., 2020). Together, these studies imply that irisin may play an important role by influencing AMPK expression during ischemic stroke. However, considering that the literature related to the neuroprotective effect of irisin and mitochondrial dynamics in stroke is limited, further research is required to confirm this role.

\section{DISCUSSION}

Although treatment strategies for stroke have been explored over several decades, intravenous thrombolysis remains the primary and most effective method (Keizman et al., 2011). However, due to the limitation presented by the short window for treating stroke, many patients are not treated in time (DiopFrimpong et al., 2011). According to a recent clinical study, the word "neuroprotection" should be replaced with "brain cytoprotection" because stroke affects the entire neurovascular unit and the underlying white matter (Savitz et al., 2019). Therefore, developing brain cytoprotectants in the context of thrombolysis along with pre-hospital/in-hospital/postthrombolysis tactics is necessary. Alternative treatment strategies such as in-hospital pre-thrombectomy cytoprotection, as well as drugs targeting the ischemic cascade within neurons and the entire neurovascular unit to limit and prevent the expansion of the ischemic core still need to be explored (Savitz et al., 2019).

Irisin is reportedly induced by physical exercise to augment energy expenditure, according to the initial report (Boström et al., 2012). A large number of clinical and experimental investigations have subsequently confirmed that acute exercise induces the release of irisin into the blood. It should be noted that although there is still some conflicting evidence (Raschke et al., 2013; Albrecht et al., 2015), it is widely believed that irisin plays substantial roles in the pathophysiology of metabolic diseases. Moreover, irisin is not only a myokine but also an adipokine (Roca-Rivada et al., 2013). Thus, irisin may be a promising therapeutic bioactive hormone for the treatment of metabolic diseases. Recent studies have uncovered some important biological functions of irisin in other systems. For example, irisin regulates depression-like behavior (Wang and Pan, 2016). Due to the crosstalk between metabolic dysfunction and cardio-cerebrovascular diseases, the role of irisin 
in the cardio-cerebrovascular system is also a deeply studied research direction.

Irisin protects against endothelial injury and ameliorates atherosclerosis in Apo-E knockout mice (Lu et al., 2015). In the field of myocardial ischemia-reperfusion, the protective role of irisin via the regulation of the SOD2 and AMPK pathways has been demonstrated (Wang et al., 2018; Xin et al., 2020). Notably, AMPK, as the main transcription factor, is very important for the crosstalk between metabolic and cardio-cerebrovascular diseases. Consequently, irisin may exert a brain cytoprotective role by influencing the expression of AMPK.

Many scholars have also suggested that irisin is an exerciseinduced muscle factor, with exercise promoting its large-scale expression in skeletal muscles, the heart, and the brain. These brain regions include Purkinje cells in the cerebellum (VarelaRodríguez et al., 2016), astrocytes in the hippocampus (Piya et al., 2014), neurons in the brain (Wang et al., 2018), the hypothalamus (Dun et al., 2013), and even the CSF (Aydin et al., 2013). Irisin is therefore suggested to have a positive impact on the nervous system. In 2017, Li et al. first provided evidence that irisin is a neuroprotective hormone in cerebral ischemia, with its expression underlying the neuroprotective effects of physical exercise against cerebral ischemia. This finding provided strong evidence that irisin may exert a brain cytoprotective role during ischemic stroke.

Evidence suggests that irisin levels are affected by a large number of stressors. It is well-established that acute exercise increases the levels of blood irisin (Jedrychowski et al., 2015;

\section{REFERENCES}

Albrecht, E., Norheim, F., Thiede, B., Holen, T., Ohashi, T., Schering, L., et al. (2015). Irisin - a myth rather than an exercise-inducible myokine. Sci. Rep. 5:8889. doi: 10.1038/srep08889

Anzell, A. R., Maizy, R., Przyklenk, K., and Sanderson, T. H. (2018). Mitochondrial quality control and disease: insights into ischemia-reperfusion injury. Mol. Neurobiol. 55, 2547-2564. doi: 10.1007/s12035-017-0503-9

Asadi, Y., Gorjipour, F., Behrouzifar, S., and Vakili, A. (2018). Irisin peptide protects brain against ischemic injury through reducing apoptosis and enhancing BDNF in a rodent model of stroke. Neurochem. Res. 43, 1549-1560. doi: 10.1007/s11064-018-2569-9

Aydin, S., Aydin, S., Kuloglu, T., Yilmaz, M., Kalayci, M., Sahin, I., et al. (2013). Alterations of irisin concentrations in saliva and serum of obese and normalweight subjects, before and after $45 \mathrm{~min}$ of a Turkish bath or running. Peptides 50, 13-18. doi: 10.1016/j.peptides.2013.09.011

Aydin, S., Kuloglu, T., Aydin, S., Eren, M. N., Celik, A., Yilmaz, M., et al. (2014). Cardiac, skeletal muscle and serum irisin responses to with or without water exercise in young and old male rats: cardiac muscle produces more irisin than skeletal muscle. Peptides 52, 68-73. doi: 10.1016/j.peptides.2013. 11.024

Bashar, S. M., Samir El-Sherbeiny, S. M., and Boraie, M. Z. (2018). Correlation between the blood level of irisin and the severity of acute myocardial infarction in exercise-trained rats. J. Basic Clin. Physiol. Pharmacol. 30, 59-71. doi: 10.1515/jbcpp-2018-0090

Berchtold, N. C., Chinn, G., Chou, M., Kesslak, J. P., and Cotman, C. W. (2005). Exercise primes a molecular memory for brain-derived neurotrophic factor protein induction in the rat hippocampus. Neuroscience 133, 853-861. doi: 10.1016/j.neuroscience.2005.03.026

Bi, J., Zhang, J., Ren, Y., Du, Z., Li, Q., Wang, Y., et al. (2019). Irisin alleviates liver ischemia-reperfusion injury by inhibiting excessive mitochondrial fission,
Löffler et al., 2015; Samy et al., 2015). Two independent studies have demonstrated that serum irisin levels decrease between 1 and $24 \mathrm{~h}$ after heart ischemia in a mouse model (Bashar et al., 2018; Zhao et al., 2019). Another study has found that plasma irisin also decreases after ischemic stroke (Li et al., 2017), which suggests that the release of irisin from muscles into the blood is inhibited after ischemic stroke. Consistent with this perspective, levels of FNDC5, the precursor of irisin, are also decreased in skeletal muscles during cerebral ischemia. However, there is no literature exploring how ischemia affects FNDC5 expression and irisin secretion from skeletal muscles, and studies are just beginning to explore the potential mechanisms involved. Because of the limited literature about the role of irisin in ischemic stroke, further studies must be conducted in the future to elucidate the potential mechanism by which irisin confers its protective effect in stroke.

\section{AUTHOR CONTRIBUTIONS}

All authors listed have made a substantial, direct and intellectual contribution to the work, and approved it for publication.

\section{ACKNOWLEDGMENTS}

Thanks to $\mathrm{CZ}$ for providing advice and direction on how to complete this review. As for preparation, YC, CM, and JG spared no effect to look for the related literature. promoting mitochondrial biogenesis and decreasing oxidative stress. Redox Biol. 20, 296-306. doi: 10.1016/j.redox.2018.10.019

Boström, P., Wu, J., Jedrychowski, M. P., Korde, A., Ye, L., Lo, J. C., et al. (2012). A PGC1- $\alpha$-dependent myokine that drives brown-fat-like development of white fat and thermogenesis. Nature 481, 463-468. doi: 10.1038/nature10777

Boyne, P., Meyrose, C., Westover, J., Whitesel, D., Hatter, K., Reisman, D. S., et al. (2019). Exercise intensity affects acute neurotrophic and neurophysiological responses poststroke. J. Appl. Physiol. 126, 431-443. doi: 10.1152/japplphysiol.00594.2018

Chen, K., Xu, Z., Liu, Y., Wang, Z., Li, Y., Xu, X., et al. (2017). Irisin protects mitochondria function during pulmonary ischemia/reperfusion injury. Sci. Transl. Med. 9:eaaao6298. doi: 10.1126/scitranslmed.aao6298

Chen, S. D., Lin, T. K., Yang, D. I., Lee, S. Y., Shaw, F. Z., Liou, C. W., et al. (2015). Roles of PTEN-induced putative kinase 1 and dynamin-related protein 1 in transient global ischemia-induced hippocampal neuronal injury. Biochem. Biophys. Res. Commun. 460, 397-403. doi: 10.1016/j.bbrc.2015.03.045

Clark, I. A., and Vissel, B. (2019). Neurodegenerative disease treatments by direct TNF reduction, SB623 cells, maraviroc and irisin and MCC950, from an inflammatory perspective - a commentary. Expert Rev. Neurother. 19, 535-543. doi: 10.1080/14737175.2019.1618710

Conti, E., Grana, D., Stefanoni, G., Corsini, A., Botta, M., Magni, P., et al. (2019). Irisin and BDNF serum levels and behavioral disturbances in Alzheimer's disease. Neurol. Sci. 40, 1145-1150. doi: 10.1007/s10072-019-03781-y

Cornelissen, V. A., and Smart, N. A. (2013). Exercise training for blood pressure: a systematic review and meta-analysis. J. Am. Heart Assoc. 2:e004473. doi: 10.1161/JAHA.112.004473

Croll, S. D., Suri, C., Compton, D. L., Simmons, M. V., Yancopoulos, G. D., Lindsay, R. M., et al. (1999). Brain-derived neurotrophic factor transgenic mice exhibit passive avoidance deficits, increased seizure severity and in vitro hyperexcitability in the hippocampus and entorhinal cortex. Neuroscience 93, 1491-1506. doi: 10.1016/S0306-4522(99)00296-1 
Ding, Y. H., Young, C. N., Luan, X., Li, J., Rafols, J. A., Clark, J. C., et al. (2005). Exercise preconditioning ameliorates inflammatory injury in ischemic rats during reperfusion. Acta Neuropathol. 109, 237-246. doi: 10.1007/s00401-004-0943-y

Diop-Frimpong, B., Chauhan, V. P., Krane, S., Boucher, Y., and Jain, R. K. (2011). Losartan inhibits collagen I synthesis and improves the distribution and efficacy of nanotherapeutics in tumors. Proc. Natl. Acad. Sci. U.S.A. 108, 2909-2914. doi: 10.1073/pnas.1018892108

Dun, S. L., Lyu, R. M., Chen, Y. H., Chang, J. K., Luo, J. J., and Dun, N. J. (2013). Irisin-immunoreactivity in neural and non-neural cells of the rodent. Neuroscience 240, 155-162. doi: 10.1016/j.neuroscience.2013.02.050

Erickson, H. P. (2013). Irisin and FNDC5 in retrospect: an exercise hormone or a transmembrane receptor? Adipocyte 2, 289-293. doi: 10.4161/adip.26082

Fan, J., Zhu, Q., Wu, Z., Ding, J., Qin, S., Liu, H., et al. (2020). Protective effects of irisin on hypoxia-reoxygenation injury in hyperglycemia-treated cardiomyocytes: role of AMPK pathway and mitochondrial protection. J. Cell. Physiol. 235, 1165-1174. doi: 10.1002/jcp.29030

Febbraio, M. A., and Pedersen, B. K. (2005). Contraction-induced myokine production and release: is skeletal muscle an endocrine organ? Exerc. Sport Sci. Rev. 33, 114-119. doi: 10.1097/00003677-200507000-00003

Flippo, K. H., Gnanasekaran, A., Perkins, G. A., Ajmal, A., Merrill, R. A., Dickey, A. S., et al. (2018). AKAP1 protects from cerebral ischemic stroke by inhibiting Drp1-dependent mitochondrial fission. J. Neurosci. 38, 8233-8242. doi: 10.1523/JNEUROSCI.0649-18.2018

Guo, P., Jin, Z., Wu, H., Li, X., Ke, J., Zhang, Z., et al. (2019). Effects of irisin on the dysfunction of blood-brain barrier in rats after focal cerebral ischemia/reperfusion. Brain Behav. 9:e01425. doi: 10.1002/brb3.1425

Guo, X., Jiang, Q., Tuccitto, A., Chan, D., Alqawlaq, S., Won, G. J., et al. (2018). The AMPK-PGC-1 $\alpha$ signaling axis regulates the astrocyte glutathione system to protect against oxidative and metabolic injury. Neurobiol. Dis. 113, 59-69. doi: 10.1016/j.nbd.2018.02.004

Gusdon, A. M., Callio, J., Distefano, G., O’Doherty, R. M., Goodpaster, B. H., Coen, P. M., et al. (2017). Exercise increases mitochondrial complex I activity and DRP1 expression in the brains of aged mice. Exp. Gerontol. 90, 1-13. doi: 10.1016/j.exger.2017.01.013

He, Z., Tian, Y., Valenzuela, P. L., Huang, C., Zhao, J., Hong, P., et al. (2018). Myokine Response to High-Intensity Interval vs. resistance exercise: an individual approach. Front. Physiol. 9:1735. doi: 10.3389/fphys.2018.01735

Herzig, S., and Shaw, R. J. (2018). AMPK: guardian of metabolism and mitochondrial homeostasis. Nat. Rev. Mol. Cell Biol. 19, 121-135. doi: $10.1038 / \mathrm{nrm} .2017 .95$

Huang, H. L., Lin, C. C., Jeng, K. C., Yao, P. W., Chuang, L. T., Kuo, S. L., et al. (2012). Fresh green tea and gallic acid ameliorate oxidative stress in kainic acid-induced status epilepticus. J. Agric. Food Chem. 60, 2328-2336. doi: $10.1021 /$ jf203709q

Islam, M. R., Young, M. F., and Wrann, C. D. (2017). “The role of FNDC5/Irisin in the nervous system and as a mediator for beneficial effects of exercise on the brain," in Hormones, metabolism and the benefits of exercise, ed. B. Spiegelman (Boston, MA: Springer), 93-102. doi: 10.1007/978-3-319-72790-5_8

Jedrychowski, M. P., Wrann, C. D., Paulo, J. A., Gerber, K. K., Szpyt, J., Robinson, M. M., et al. (2015). Detection and quantitation of circulating human irisin by tandem mass spectrometry. Cell Metab. 22, 734-740. doi: 10.1016/j.cmet.2015.0 8.001

Jin, Y., Sumsuzzman, D. M., Choi, J., Kang, H., Lee, S. R., and Hong, Y. (2018). Molecular and functional interaction of the myokine irisin with physical exercise and Alzheimer's disease. Molecules 23:3329. doi: 10.3390/molecules 2312 3229

Jin, Z., Guo, P., Li, X., Ke, J., Wang, Y., and Wu, H. (2019). Neuroprotective effects of irisin against cerebral ischemia/ reperfusion injury via Notch signaling pathway. Biomed. Pharmacother. 120:109452. doi: 10.1016/j.biopha.2019.109452

Kameoka, S., Adachi, Y., Okamoto, K., Iijima, M., and Sesaki, H. (2018). Phosphatidic acid and cardiolipin coordinate mitochondrial dynamics. Trends Cell Biol. 28, 67-76. doi: 10.1016/j.tcb.2017.08.011

Karimi-Khouzani, O., Heidarian, E., and Amini, S. A. (2017). Antiinflammatory and ameliorative effects of gallic acid on fluoxetine-induced oxidative stress and liver damage in rats. Pharmacol. Rep. 69, 830-835. doi: 10.1016/j.pharep.2017.03.011

Katan, M., and Luft, A. (2018). Global burden of stroke. Semin. Neurol. 38, 208-211. doi: 10.1055/s-0038-1649503

Keizman, D., Huang, P., Eisenberger, M. A., Pili, R., Kim, J. J., Antonarakis, E. S. et al. (2011). Angiotensin system inhibitors and outcome of sunitinib treatment in patients with metastatic renal cell carcinoma: a retrospective examination. Eur. J. Cancer 47, 1955-1961. doi: 10.1016/j.ejca.2011.04.019

Khoshnam, S. E., Winlow, W., Farzaneh, M., Farbood, Y., and Moghaddam, H. F. (2017). Pathogenic mechanisms following ischemic stroke. Neurol. Sci. 38, 1167-1186. doi: 10.1007/s10072-017-2938-1

Kim, H., Wrann, C. D., Jedrychowski, M., Vidoni, S., Kitase, Y., Nagano, K., et al. (2018). Irisin mediates effects on bone and fat via $\alpha \mathrm{V}$ integrin receptors. Cell 175, 1756-1768.e17. doi: 10.1016/j.cell.2018.10.025

Lai, Y., Lin, P., Chen, M., Zhang, Y., Chen, J., Zheng, M., et al. (2020). Restoration of L-OPA1 alleviates acute ischemic stroke injury in rats via inhibiting neuronal apoptosis and preserving mitochondrial function. Redox Biol. 34:101503. doi: 10.1016/j.redox.2020.101503

Lapchak, P. A., and Zhang, J. H. (2017). The high cost of stroke and stroke cytoprotection research. Transl. Stroke Res. 8, 307-317. doi: 10.1007/s12975-016-0518-y

Lee, H. J., Lee, J. O., Kim, N., Kim, J. K., Kim, H. I., Lee, Y. W., et al. (2015). Irisin, a novel myokine, regulates glucose uptake in skeletal muscle cells via AMPK. Mol. Endocrinol. 29, 873-881. doi: 10.1210/me.2014-1353

Li, D. J., Li, Y. H., Yuan, H. B., Qu, L. F., and Wang, P. (2017). The novel exercise-induced hormone irisin protects against neuronal injury via activation of the Akt and ERK1/2 signaling pathways and contributes to the neuroprotection of physical exercise in cerebral ischemia. Metabolism 68, 31-42. doi: 10.1016/j.metabol.2016.12.003

Li, Y., and Liu, X. (2018). Novel insights into the role of mitochondrial fusion and fission in cardiomyocyte apoptosis induced by ischemia/reperfusion. J. Cell. Physiol. 233, 5589-5597. doi: 10.1002/jcp.26522

Löffler, D., Müller, U., Scheuermann, K., Friebe, D., Gesing, J., Bielitz, J., et al. (2015). Serum irisin levels are regulated by acute strenuous exercise. J. Clin. Endocrinol. Metab. 100, 1289-1299. doi: 10.1210/jc.2014-2932

Lu, J., Xiang, G., Liu, M., Mei, W., Xiang, L., and Dong, J. (2015). Irisin protects against endothelial injury and ameliorates atherosclerosis in apolipoprotein E-Null diabetic mice. Atherosclerosis 243, 438-448. doi: 10.1016/j.atherosclerosis.2015.10.020

Mang, C. S., Campbell, K. L., Ross, C. J., and Boyd, L. A. (2013). Promoting neuroplasticity for motor rehabilitation after stroke: considering the effects of aerobic exercise and genetic variation on brain-derived neurotrophic factor. Phys. Ther. 93, 1707-1716. doi: 10.2522/ptj.20130053

Matsuda, F., Sakakima, H., and Yoshida, Y. (2011). The effects of early exercise on brain damage and recovery after focal cerebral infarction in rats. Acta Physiol. 201, 275-287. doi: 10.1111/j.1748-1716.2010.02174.x

Otsuka, S., Sakakima, H., Sumizono, M., Takada, S., Terashi, T., and Yoshida, Y. (2016). The neuroprotective effects of preconditioning exercise on brain damage and neurotrophic factors after focal brain ischemia in rats. Behav. Brain Res. 303, 9-18. doi: 10.1016/j.bbr.2016.01.049

Peng, J., Deng, X., Huang, W., Yu, J. H., Wang, J. X., Wang, J. P., et al. (2017). Irisin protects against neuronal injury induced by oxygen-glucose deprivation in part depends on the inhibition of ROS-NLRP3 inflammatory signaling pathway. Mol. Immunol. 91, 185-194. doi: 10.1016/j.molimm.2017.09.014

Piya, M. K., Harte, A. L., Sivakumar, K., Tripathi, G., Voyias, P. D., James, S., et al. (2014). The identification of irisin in human cerebrospinal fluid: influence of adiposity, metabolic markers, and gestational diabetes. Am. J. Physiol. Endocrinol. Metab. 306, E512-E518. doi: 10.1152/ajpendo.00308. 2013

Quirié, A., Hervieu, M., Garnier, P., Demougeot, C., Mossiat, C., Bertrand, N., et al. (2012). Comparative effect of treadmill exercise on mature BDNF production in control versus stroke rats. PLOS ONE 7:e44218. doi: 10.1371/journal.pone.0044218

Raefsky, S. M., and Mattson, M. P. (2017). Adaptive responses of neuronal mitochondria to bioenergetic challenges: roles in neuroplasticity and disease resistance. Free Radic. Biol. Med. 102, 203-216. doi: 10.1016/j.freeradbiomed.2016.11.045 
Raschke, S., Elsen, M., Gassenhuber, H., Sommerfeld, M., Schwahn, U., Brockmann, B., et al. (2013). Evidence against a beneficial effect of irisin in humans. PLoS ONE 8:e73680. doi: 10.1371/journal.pone.0073680

Rasmussen, P., Brassard, P., Adser, H., Pedersen, M. V., Leick, L., Hart, E., et al. (2009). Evidence for a release of brain-derived neurotrophic factor from the brain during exercise. Exp. Physiol. 94, 1062-1069. doi: 10.1113/expphysiol.2009.048512

Roca-Rivada, A., Castelao, C., Senin, L. L., Landrove, M. O., Baltar, J., Belén Crujeiras, A., et al. (2013). FNDC5/irisin is not only a myokine but also an adipokine. PLoS ONE 8:e60563. doi: 10.1371/journal.pone.0060563

Ruas, J. L., White, J. P., Rao, R. R., Kleiner, S., Brannan, K. T., Harrison, B. C., et al. (2012). A PGC-1 $\alpha$ isoform induced by resistance training regulates skeletal muscle hypertrophy. Cell 151, 1319-1331. doi: 10.1016/j.cell.2012.10.050

Ryan, A. S., Li, G., Hafer-Macko, C., and Ivey, F. M. (2017). Resistive training and molecular regulators of vascular-metabolic risk in chronic stroke. J. Stroke Cerebrovasc. Dis. 26, 962-968. doi: 10.1016/j.jstrokecerebrovasdis.2016.11.003

Sakakima, H., Khan, M., Dhammu, T. S., Shunmugavel, A., Yoshida, Y., Singh, I., et al. (2012). Stimulation of functional recovery via the mechanisms of neurorepair by S-nitrosoglutathione and motor exercise in a rat model of transient cerebral ischemia and reperfusion. Restor. Neurol. Neurosci. 30, 383-396. doi: 10.3233/RNN-2012-110209

Samy, D. M., Ismail, C. A., and Nassra, R. A. (2015). Circulating irisin concentrations in rat models of thyroid dysfunction - effect of exercise. Metabolism 64, 804-813. doi: 10.1016/j.metabol.2015.01.001

Savitz, S. I., Baron, J. C., Fisher, M., and STAIRX Consortium (2019). Stroke treatment academic industry roundtable $\mathrm{X}$ : Brain cytoprotection therapies in the reperfusion era. Stroke 50, 1026-1031. doi: 10.1161/STROKEAHA.118.023927

Siteneski, A., Cunha, M. P., Lieberknecht, V., Pazini, F. L., Gruhn, K., Brocardo, P. S., et al. (2018). Central irisin administration affords antidepressant-like effect and modulates neuroplasticity-related genes in the hippocampus and prefrontal cortex of mice. Prog. Neuropsychopharmacol. Biol. Psychiatr. 84, 294-303. doi: 10.1016/j.pnpbp.2018.03.004

Tan, Y., Ouyang, H., Xiao, X., Zhong, J., and Dong, M. (2019). Irisin ameliorates septic cardiomyopathy via inhibiting DRP1-related mitochondrial fission and normalizing the JNK-LATS2 signaling pathway. Cell Stress Chaperones 24, 595-608. doi: 10.1007/s12192-019-00992-2

Tang, H., Yu, R., Liu, S., Huwatibieke, B., Li, Z., and Zhang, W. (2016). Irisin inhibits hepatic cholesterol synthesis via AMPK-SREBP2 signaling. EBioMed. Lancet 6, 139-148. doi: 10.1016/j.ebiom.2016.02.041

Trewin, A. J., Berry, B. J., and Wojtovich, A. P. (2018). Exercise and mitochondrial dynamics: keeping in shape with ROS and AMPK. Antioxidants 7:7. doi: $10.3390 /$ antiox7010007

Tu, T., Peng, J., and Jiang, Y. (2020). FNDC5/Irisin: a new protagonist in acute brain injury. Stem Cells Dev. 29, 533-543. doi: 10.1089/scd.2019.0232

Varela-Rodríguez, B. M., Pena-Bello, L., Juiz-Valiña, P., Vidal-Bretal, B., Cordido, F., and Sangiao-Alvarellos, S. (2016). FNDC5 expression and circulating irisin levels are modified by diet and hormonal conditions in hypothalamus, adipose tissue and muscle. Sci. Rep. 6:29898. doi: 10.1038/srep29898

Wahl, P., Jansen, F., Achtzehn, S., Schmitz, T., Bloch, W., Mester, J., et al. (2014). Effects of high intensity training and high volume training on endothelial microparticles and angiogenic growth factors. PLOS ONE 9:e96024. doi: 10.1371/journal.pone.0096024

Wang, C., and Youle, R. (2016). Cell biology: form follows function for mitochondria. Nature 530, 288-289. doi: 10.1038/530288a

Wang, K., Li, H., Wang, H., Wang, J. H., Song, F., and Sun, Y. (2018). Irisin exerts neuroprotective effects on cultured neurons by regulating astrocytes. Mediators Inflam. 2018:9070341. doi: 10.1155/2018/9070341

Wang, K., Liu, Z., Zhao, M., Zhang, F., Wang, K., Feng, N., et al. (2020). Kappa-opioid receptor activation promotes mitochondrial fusion and enhances myocardial resistance to ischemia and reperfusion injury via STAT3-OPA1 pathway. Eur. J. Pharmacol. 874:172987. doi: 10.1016/j.ejphar.2020.172987

Wang, S., and Pan, J. (2016). Irisin ameliorates depressive-like behaviors in rats by regulating energy metabolism. Biochem. Biophys. Res. Commun. 474, 22-28. doi: $10.1016 /$ j.bbrc.2016.04.047
Wang, Z., Chen, K., Han, Y., Zhu, H., Zhou, X., Tan, T., et al. (2018). Irisin protects heart against ischemia-reperfusion injury through a SOD2dependent mitochondria mechanism. J. Cardiovasc. Pharmacol. 72, 259-269. doi: 10.1097/FJC.0000000000000608

Wei, N., Pu, Y., Yang, Z., Pan, Y., and Liu, L. (2019). Therapeutic effects of melatonin on cerebral ischemia reperfusion injury: role of Yap-OPA1 signaling pathway and mitochondrial fusion. Biomed. Pharmacother. 110, 203-212. doi: 10.1016/j.biopha.2018.11.060

Wrann, C. D., White, J. P., Salogiannnis, J., Laznik-Bogoslavski, D., Wu, J., Ma, D., et al. (2013). Exercise induces hippocampal BDNF through a PGC-1 $\alpha$ /FNDC5 pathway. Cell Metab. 18, 649-659. doi: 10.1016/j.cmet.2013. 09.008

Xin, C., Zhang, Z., Gao, G., Ding, L., Yang, C., Wang, C., et al. (2020). Irisin attenuates myocardial ischemia/reperfusion injury and improves mitochondrial function through AMPK pathway in diabetic mice. Front. Pharmacol. 11:565160. doi: 10.3389/fphar.2020.565160

$\mathrm{Xu}$, B. (2013). BDNF (I)rising from exercise. Cell Metab. 18, 612-614. doi: 10.1016/j.cmet.2013.10.008

Yang, J. P., Liu, H. J., and Liu, X. F. (2010). VEGF promotes angiogenesis and functional recovery in stroke rats. J. Invest. Surg. 23, 149-155. doi: 10.3109/08941930903469482

Yau, S. Y., Li, A., Xu, A., and So, K. F. (2015). Fat cell-secreted adiponectin mediates physical exercise-induced hippocampal neurogenesis: an alternative anti-depressive treatment? Neural Regen. Res. 10, 7-9. doi: 10.4103/1673-5374.150637

Young, M. F., Valaris, S., and Wrann, C. D. (2019). A role for FNDC5/Irisin in the beneficial effects of exercise on the brain and in neurodegenerative diseases. Prog. Cardiovasc. Dis. 62, 172-178. doi: 10.1016/j.pcad.2019.02.007

Yu, Q., Li, G., Ding, Q., Tao, L., Li, J., Sun, L., et al. (2020). Irisin protects brain against ischemia/reperfusion injury through suppressing TLR4/MyD88 pathway. Cerebrovasc. Dis. (Basel, Switzerland) 49, 346-354. doi: 10.1159/000505961

Zabala, V., Boylan, J. M., Thevenot, P., Frank, A., Senthoor, D., Iyengar, V., et al. (2019). Transcriptional changes during hepatic ischemia-reperfusion in the rat. PLoS ONE 14:e0227038. doi: 10.1371/journal.pone.0227038

Zhang, B., Zhang, H. X., Shi, S. T., Bai, Y. L., Zhe, X., Zhang, S. J., et al. (2019). Interleukin-11 treatment protected against cerebral ischemia/reperfusion injury. Biomed. Pharmacother. 115:108816. doi: 10.1016/j.biopha.2019.108816

Zhang, L., He, Z., Zhang, Q., Wu, Y., Yang, X., Niu, W., et al. (2014). Exercise pretreatment promotes mitochondrial dynamic protein OPA1 expression after cerebral ischemia in rats. Int. J. Mol. Sci. 15, 4453-4463. doi: 10.3390/ijms15034453

Zhang, R., Ji, J., Zhou, X., and Li, R. (2020). Irisin pretreatment protects kidneys against acute kidney injury induced by ischemia/reperfusion via upregulating the expression of uncoupling protein 2. BioMed Res. Int. 2020:6537371. doi: 10.1155/2020/6537371

Zhang, Z. G., Zhang, L., Jiang, Q., Zhang, R., Davies, K., Powers, C., et al. (2000). VEGF enhances angiogenesis and promotes blood-brain barrier leakage in the ischemic brain. J. Clin. Invest. 106, 829-838. doi: 10.1172/JCI9369

Zhao, Y. T., Wang, J., Yano, N., Zhang, L. X., Wang, H., Zhang, S., et al. (2019). Irisin promotes cardiac progenitor cell-induced myocardial repair and functional improvement in infarcted heart. J. Cell. Physiol. 234, 1671-1681. doi: $10.1002 /$ jcp. 27037

Conflict of Interest: The authors declare that the research was conducted in the absence of any commercial or financial relationships that could be construed as a potential conflict of interest.

Copyright (c) $2020 \mathrm{Liu}, \mathrm{Zhu}, \mathrm{Guo}$, Chen and Meng. This is an open-access article distributed under the terms of the Creative Commons Attribution License (CC BY). The use, distribution or reproduction in other forums is permitted, provided the original author(s) and the copyright owner(s) are credited and that the original publication in this journal is cited, in accordance with accepted academic practice. No use, distribution or reproduction is permitted which does not comply with these terms. 